\title{
Synthesis and Characterization of Exfoliated Graphite/ABS Composites
}

\author{
Bhardwaj Neha, K. S. Manjula, B. Srinivasulu, Shit C. Subhas* \\ Central Institute of Plastics Engineering and Technology, Ahmedabad, India. \\ Email: "subhasch.shit@yahoo.com
}

Received June 14, 2012; revised July 25, 2012; accepted August 10, 2012

\begin{abstract}
Exfoliated graphite was prepared by chemical route and then further subjected to thermal oxidation and sonication for size reduction and increased interlayer spacing in natural flake graphite. Exfoliated graphite/ABS composites were prepared with varying filler concentration by solution casting method. Exfoliated graphite and composites were characterised by scanning electron microscopy and energy dispersive X-ray analysis, Fourier transform infrared spectroscopy and X-ray diffractometry techniques. After thermal exfoliation significant peak of graphite at $2 \theta=26.4^{\circ}$ disappeared completely, confirming successful exfoliation of graphite. SEM images revealed homogeneous dispersion of exfoliated graphite in the matrix and EDAX confirmed successful reduction of graphite oxide.
\end{abstract}

Keywords: Graphite Intercalated Compound; Exfoliated Graphite; Exfoliated Graphite/ABS Composites; Scanning Electron Microscopy; X-Ray Diffractometry

\section{Introduction}

Exfoliated graphite has received attention as filler in composites in recent times due to prominent properties it imparts to the host polymeric matrices. These special attributes make the resulting composites suitable in certain areas of application.

Exfoliated graphite/polymer composites are finding applications in varied areas such as charge storage devices, electronics packaging, electromagnetic interfereence shielding and antistatic coatings, conducting packaging; majorly because of the conducting nature of graphite [1]. As reported by Toyota researchers [2-4], upon addition of layered silicates, properties such as mechani$\mathrm{cal}$, thermal, and barrier performance are improved in intercalated or delaminated composites in comparison to conventional composites. Natural flake graphite has properties similar to layered silicates which can be treatment for improvement of electrical conductivity and other properties mentioned above.

The graphite crystal lattice consists of graphene layers or $2 \mathrm{~d}$ sheet formed by $s p^{2}$ hybridized carbon atoms. The carbon sheets are bounded by weak Van Der Waals forces and the $\mathrm{d}$ spacing between layers is $3.37 \AA$ [5]. Graphite is a good electrical conductor with an electrical conductivity in the range of $10^{4} \mathrm{~S} / \mathrm{m}$ at room temperature [6]. Natural graphite needs to be treated as it is not suit-

\footnotetext{
"Corresponding author.
}

able for use in its natural form. This happens because of graphite particle size is on millimetre scale which leads to lower aspect ratio [7] and can be increased by reducing its particle size.

The weak Van Der Waals interactions among graphene layers are present, intercalating agents like sulphuric acid and nitric acid, can penetrate into the layers of graphite by diffusion through edge planes to form layered crystals [8]. The resulting products composed of guest intercalant and host graphite referred to as graphite intercalation compounds (GICs) [8-10]. As reviewed by Daniel et al. [9], flake graphite has complex structure and inherent localized defects in its $p$-structure that may serve as seed points for the oxidation process. The use of nitric acid and Potassium chlorate are the strongest oxidation technique used on a preparative scale which results in better oxidation than use of intercalating agent alone.

In the present investigation, exfoliated graphite, exfoliated graphite/ABS composites were synthesised and characterised by SEM, EDAX, FTIR, XRD and electrical conductivity.

\section{Experimental}

\subsection{Materials}

Graphite fine powder with carbon content $98 \%$ and particle size of 60 mesh (Loba Chemi Pvt. Ltd.), conc. sul- 
phuric acid $\left(\mathrm{H}_{2} \mathrm{SO}_{4}, 98 \%\right)$, nitric acid $\left(\mathrm{HNO}_{3}, 95 \%\right)$, potassium hydroxide $(\mathrm{KOH})$, hydrochloric acid $(\mathrm{HCl})$, potassium chlorate $\left(\mathrm{KClO}_{3}\right)$, tetrahydrofuran (THF) (Merck) and ABS grade 920 (natural colour) supplied by INEOS ABS Vadodara, Gujarat (India).

\subsection{Synthesis of Exfoliated Graphite}

Synthesis of exfoliated graphite involves chemical treatment of thermal exfoliation for the formulation and reduction of graphite oxide.

\subsubsection{Intercalation and Oxidation of Natural Flake Graphite}

In the first step, the chemical route (Staudenmaier method) was used for preparation of graphite oxide (GO) $[11,12]$. In this method, a three necked round bottom flask $(500 \mathrm{ml})$ was purged with nitrogen and immersed in an ice bath. Homogeneous mixture of conc. $\mathrm{HNO}_{3}$ and conc. $\mathrm{H}_{2} \mathrm{SO}_{4}$ in $1: 2 \mathrm{v} / \mathrm{v}$ was prepared and $20 \mathrm{~g}$ Graphite added slowly to minimize the risk of explosion in an ice bath under vigorous stirring. Graphite intercalated compound (GIC) formed after treating natural flake graphite with sulphuric acid and nitric acid and was further oxidised to give graphite oxide (GO). $165 \mathrm{~g}$ of Potassium chlorate was added very slowly to minimize the risk of explosion as reaction was hazardous. The reaction was then continued for $120 \mathrm{hr}$ at room temperature. The resultant slurry was filtered through whatman filter paper and washed with excess deionised water and $5 \% \mathrm{HCl}$ solution to remove left-over sulphate ions $\left(\mathrm{SO}_{4}{ }^{-2}\right)$. The resulting GO solution was neutralised with potassium hydroxide solution and washed with distilled water. The powder was dried in an oven for $48 \mathrm{hr}$ at $135^{\circ} \mathrm{C}$.

\subsubsection{Reduction of Graphite Oxide (GO)}

The second step involved reduction of GO which was carried out to increase interlayer spacing and further reduction in Van Der Waals forces. This can be accomplished by various methods such as chemical functionalization [13], electrochemical reduction or by thermal exfoliation as explained here.
The dried GO powder was quickly inserted into a preheated muffle furnace temperature at $950^{\circ} \mathrm{C}$ and held there for 30 seconds. The temperature of $950^{\circ} \mathrm{C}$ and above has been found to be adequate for thermal exfoliation and in situ reduction of GO [6]. The acid molecules residing in the inter-graphene spacing are decomposed at such a high temperature, generating volatile gas molecules that could push apart graphene planes [14,15]. Exfoliation of the stacked structure occurs through the emission of carbon dioxide generated by heating GO to such a high temperature [9]. Schematic illustration of the synthesis of exfoliated graphite is shown in Figure 1.

\subsection{Preparation Exfoliated Graphite-ABS Composite Films}

Tetrahydrofuran (THF) was used as the dispersion medium for exfoliated graphite for further separation of layers by sonication for duration of 30 minutes in a probe sonicator at frequency of $35 \mathrm{kHz}$ [8]. $1 \mathrm{~g}$ of ABS was dissolved in $10 \mathrm{ml}$ of THF and then added to sonicated exfoliated graphite-THF dispersion. The solution was again subjected to probe sonication for duration of 30 min for uniform dispersion into polymeric matrix. After, the sonication solution was poured into a predried greased petridish and left undisturbed at room temperature for a period of $48 \mathrm{hr}$. The average thickness of cast film was $0.27 \mathrm{~mm}$. The film was kept in the oven for a period of $24 \mathrm{hr}$ at $80^{\circ} \mathrm{C}$ to remove possible traces of solvent that may still be present. Composite films were prepared by same methodology with varying exfoliated graphite concentration such as $2 \mathrm{wt} \%, 4 \mathrm{wt} \%, 8 \mathrm{wt} \%$, $12.7 \mathrm{wt} \%, 17.5 \mathrm{wt} \%$.

\subsection{Specimen Characterization}

The microstructure of the films was characterised by scanning electron microscopy with EDAX attachment. To carry out SEM and EDAX analysis samples of natural flake graphite, exfoliated graphite and Exfoliated graphite/ABS composite films were coated with gold in Bal-Tec SCD 500 sputter coater and examined under (FEI ESEM Quanta (EDAX) machine set up. FTIR

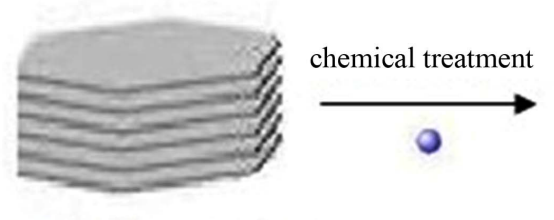

natural flake graphite with inter graphene spacing of $<0.34 \mathrm{~nm}$

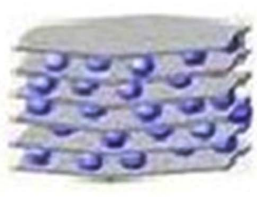

thermal exfoliation

$\mathrm{GIC}(\mathrm{GO})$

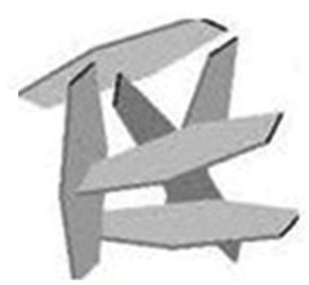

exfoliated graphite with interplane spacing $>20$ $\mathrm{nm}$ to several $\mu \mathrm{m}$

Figure 1. Schematic illustration of the synthesis of exfoliated graphite. 
spectra were obtained on Bruker Alpha Spectrometer. XRD was performed on Bruker D8 Advance in the range of 0 to $90^{\circ}$. Electrical conductivity was measured with a Kiethley 2410 two-probe electrometer. To provide better contact with measuring probe surface, the samples were coated with silver paint and bonded with copper connecting wires.

\section{Results and Discussion}

\subsection{SEM Analysis of Natural Graphite and Exfoliated Graphite}

The SEM micrographs of natural flake graphite and exfoliated graphite are shown in Figure 2. Figures 2(a) and (b) show agglomerates of graphite under $800 \mathrm{x}$ and 3000x magnifications. Figures 2(c) and (d) are for exfoliated graphite after thermal reduction of the graphite oxide. The SEM micrographs in Figure 2(c) clearly revealed exfoliation of GO at $1000 \times$ magnification while Figure 2(d) showed close view of separated sheets at the much higher magnification of $12000 \times$.

EDAX analysis of exfoliated graphite is as shown in Figure 3, which reveals graphite oxide reduction to exfoliated graphite as no traces of oxygen was found.

\subsection{Morphological Analysis of Composite Films}

Figures 4(a)-(d) show SEM micrographs of composite films with varied filler concentration of $2 \mathrm{wt} \%$ (volume fraction $\phi=0.0049$ ) at $8000 \times$ magnification, (b) $8 \mathrm{wt} \%$ $(\phi=0.07)$ at $1000 \times$ magnification, (c) $12.5 \mathrm{wt} \%(\phi=$ $0.111)$ at $200 \times$ magnification (d) $17.5 \mathrm{wt} \%(\phi=0.148)$ at $400 \times$ magnification. As revealed by the micrographs, the exfoliated graphite dispersion was found to be homogeneous within the ABS matrix

\subsection{Ftir Analysis}

The spectra of $2 \mathrm{wt} \%$ and $17.5 \mathrm{wt} \%$ are as shown in Figures 5(a) and (b) respectively. It was observed that the band near $1500 \mathrm{~cm}^{-1}-1550 \mathrm{~cm}^{-1}$ region corresponding to the aromatic $\mathrm{C}=\mathrm{C}$ stretching vibration and peak became broad on increasing graphite content $[16,17]$.

The IR spectrum of exfoliated graphite/ABS composite for volume fraction of 0.175 and 0.0049 shows characteristic peaks for three components of ABS. The band at $2237 \mathrm{~cm}^{-1}$ is attributable to the $-\mathrm{CN}$ band of acrylonitrile component, the band corresponding to aromatic ring of styrene component at 1602, 1494, 761 and $699 \mathrm{~cm}^{-1}$ and the presence of band at $967 \mathrm{~cm}^{-1}$ which is due to butadiene (trans) and the double bond of butadiene component appeared at $911 \mathrm{~cm}^{-1}[18]$.

\subsection{X-Ray Diffractometry}

The XRD spectra of pristine graphite, exfoliated graphite and $2 \mathrm{wt} \%$ exfoliated graphite/ABS composite as shown in Figures 6(a)-(c) respectively. The strong and sharp diffraction peak of pristine graphite visible at $2 \theta=26.4^{\circ}$ corresponds to a d-spacing of $3.37 \AA$, which completely disappears after thermal exfoliation of the graphite oxide (GO). No apparent diffraction peak detected, which means that the periodic structure of GO was eliminated and size of graphite was thus reduced [5].

\subsection{Conducting Properties of Composites}

Electrical resistivity of composite film samples including neat ABS film are reported in Table 1. It is evident from the results that there is gradual decrease in resistivity value as the concentration of exfoliated graphite increases in the composites. Electrical resistivity value for neat ABS falls in insulating range, while composite with $17.5 \mathrm{wt} \%$ of exfoliated graphite was found to be moderately conducting due to the formation of conducting network with in the polymeric matrix $[6,7]$.

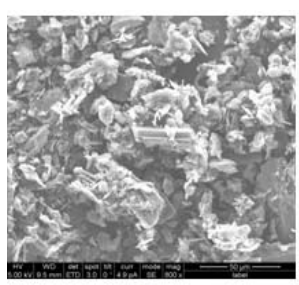

(a)

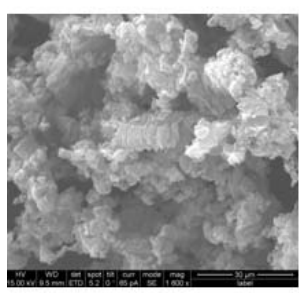

(c)

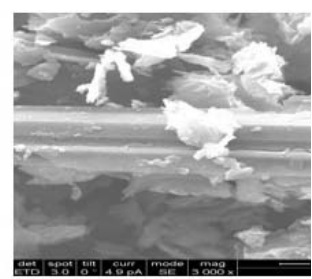

(b)

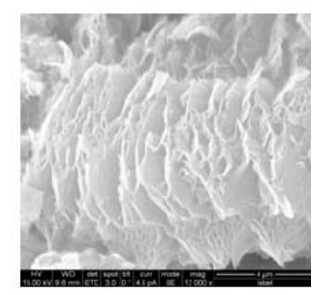

(d)
Figure 2. (a) The SEM micrographs of natural flake graphite

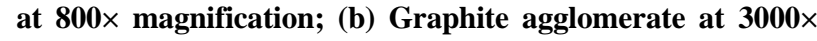
magnification; (c) Exfoliated graphite at 1000× magnification and (d) Separated sheets of exfoliated graphite at 12000 $\times$ magnification.

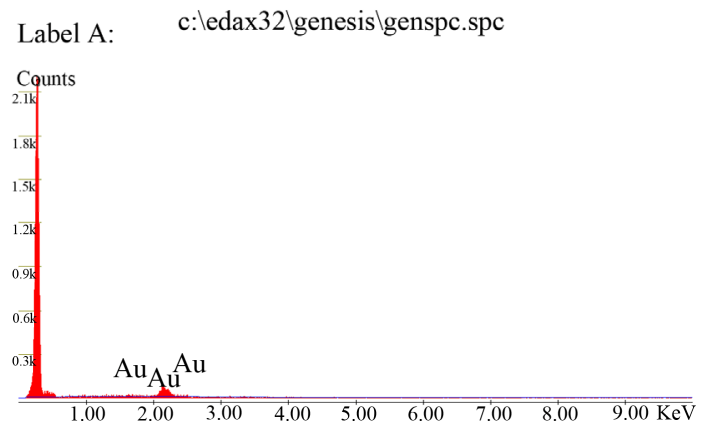

Figure 3. Elemental composition of exfoliated graphite powder. 


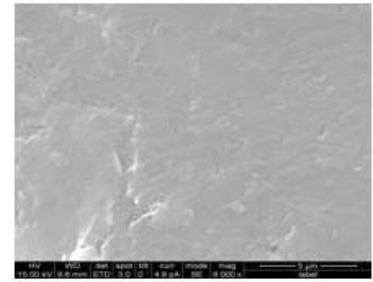

(a)

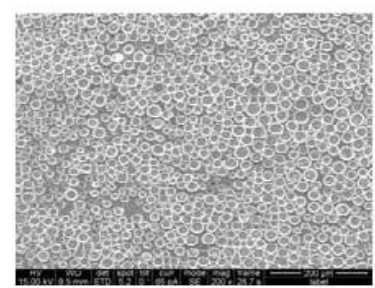

(c)

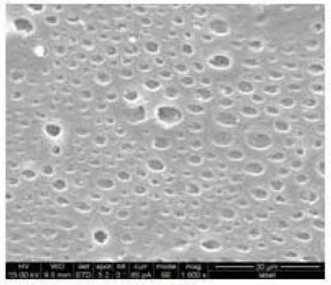

(b)

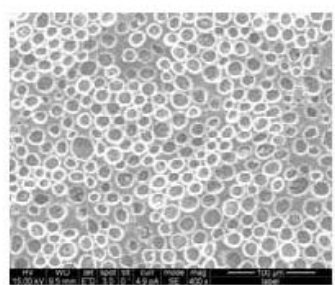

(d)
Figure 4. The SEM micrographs of the composite film for (a) $2 \mathrm{wt} \%$ at $3000 \times$ magnification; (b) $8 \mathrm{wt} \%$ at $1000 \times$ magnification; (c) $12.7 \mathrm{wt} \%$ at $200 \times$ magnification; (d) $17.5 \mathrm{wt} \%$ at $400 \times$ magnification.

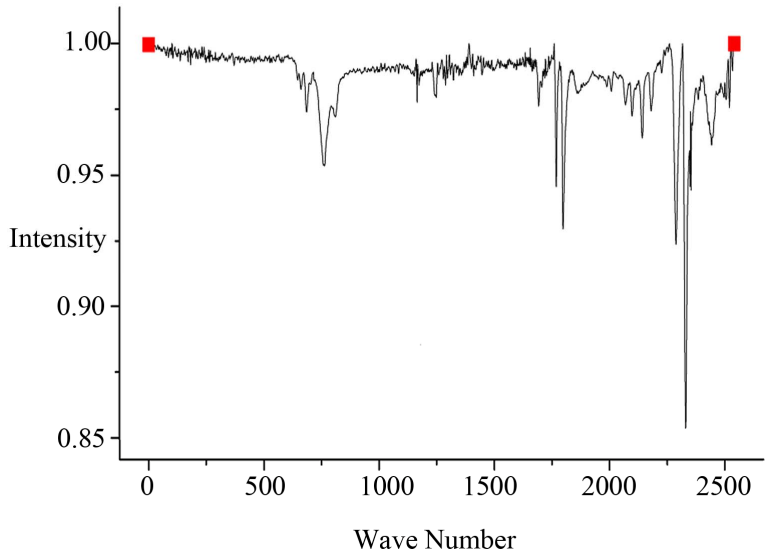

(a)

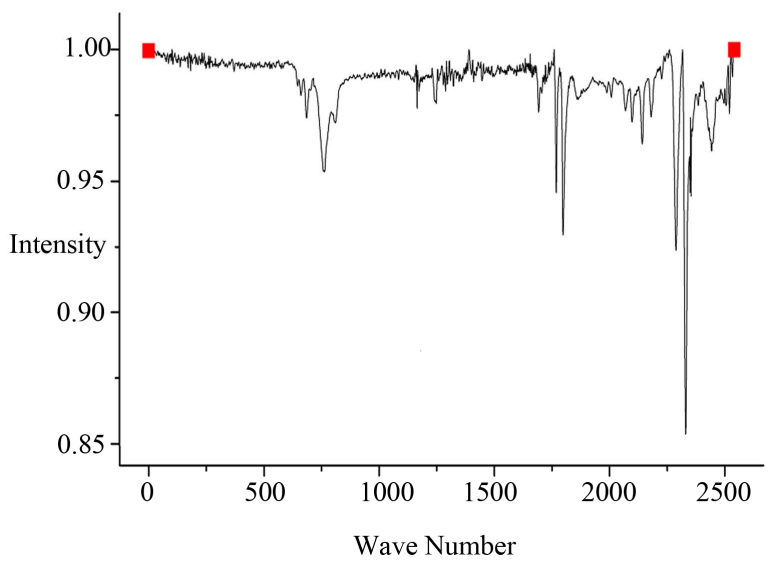

(b)

Figure 5. (a) FTIR spectrum of exfoliated graphite (2 wt \%)/ ABS composite; (b) FTIR spectrum of exfoliated graphite (17.5 wt\%)/ABS composite.

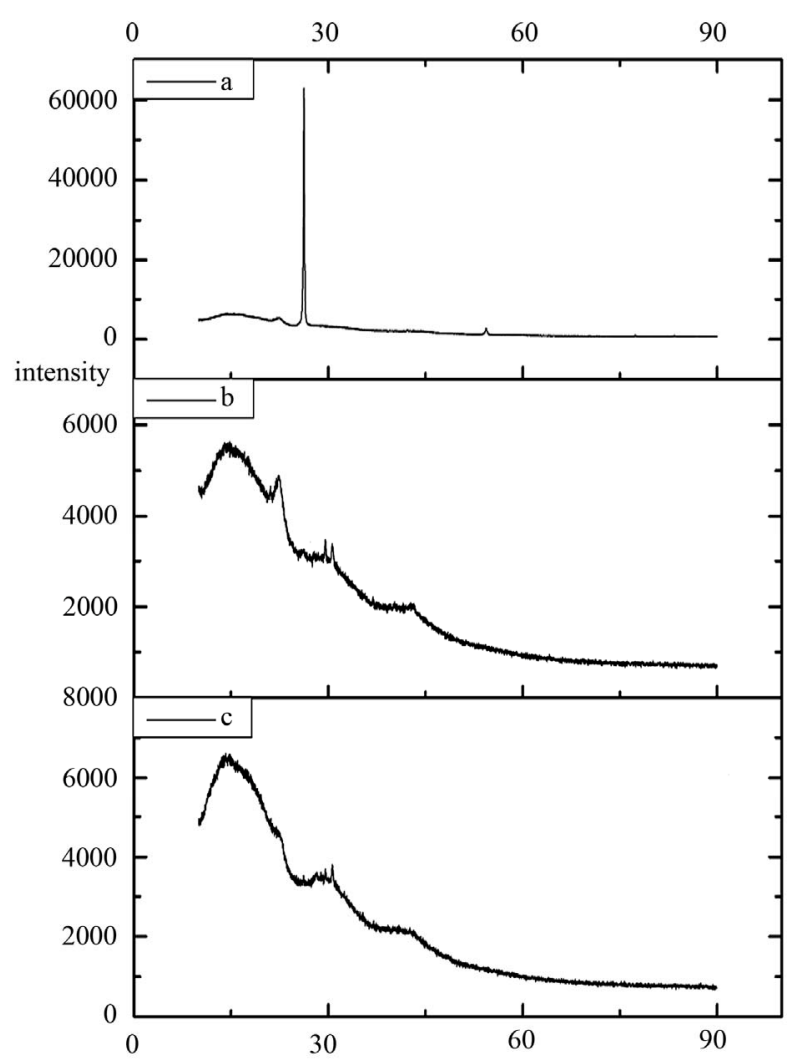

Figure 6. XRD spectra of (a) natural flake graphite, (b) exfoliated graphite and (c) exfoliated graphite (2 wt\%)/ABS composite film.

Table 1. Conducting properties of exfoliated graphite/ABS composites

\begin{tabular}{ccc}
\hline $\begin{array}{c}\text { Sample } \\
\text { Code }\end{array}$ & $\begin{array}{c}\text { Weight percentage of } \\
\text { exfoliated graphite }(\%)\end{array}$ & $\begin{array}{c}\text { Resistivity } \\
(\Omega \mathrm{cm})\end{array}$ \\
\hline EG/ABS 0 & 0 & $1.1 \times 10^{12}$ \\
EG/ABS 2 & 2 & $1.32 \times 10^{9}$ \\
EG/ABS 3 & 4 & $2.01 \times 10^{9}$ \\
EG/ABS 4 & 8 & $1.02 \times 10^{8}$ \\
EG/ABS 5 & 12.5 & $2.13 \times 10^{7}$ \\
EG/ABS 6 & 17.5 & $1.12 \times 10^{5}$ \\
\hline
\end{tabular}

\section{Conclusion}

Exfoliated graphite was successfully obtained through thermal exfoliation reduction reaction which was confirmed by SEM and EDAX analysis. Treatment of the natural flake graphite resulted in size reduction and increase in interlayer spacing of exfoliated graphite as confirmed by XRD. Uniform dispersion of exfoliated graphite was achieved by sonication even at higher filler concentration as revealed by SEM. Incorporation of exfoliated graphite decreases the electrical resistivity value of the composites. 


\section{REFERENCES}

[1] V. Panwar, B. Kang, J. O. Park, S. Park and R. M. Mehta, "Study of Dielectric Properties Of Styrene-AcryloniTrile Graphite Sheets Composites In Low And High Frequency Region," European Polymer Journal, Vol. 45, No. 6, 2009, pp. 1777-1784. doi:10.1016/j.eurpolymj.2009.02.020

[2] R. A. Vaia, K. D. Jandt, E. J. Kramer and E. P. Giannelis, "Kinetics of Polymer Melt Intercalation," Macromolecules, Vol. 28, No. 24, 1995, pp. 8080-8085. doi:10.1021/ma00128a016

[3] E. Manias, A. Touny, L. Wu, K. Strawhecher, B. Lu and T. C. Chung, "Polypropylene/Montmorillonite Nanocomposites. Review of the Synthetic Routes and Materials Properties," Chemistry of Materials, Vol. 13, No. 10, 2001, pp. 3516-3523. doi:10.1021/cm0110627

[4] M. Okamoto, P. H. Nam, P. Maiti, T. Kotaka, T. Nakayama, M. Takada, M. Ohshima, A. Usuki, N. Hasegawa and H. Okamoto, "Biaxial Flow-Induced Alignment of Silicate Layers in Polypropylene/Clay Nanocomposite Foam," Nano Letters, Vol. 1, No. 9, 2001, pp. 503-505. doi:10.1021/n1010051+

[5] A. Yasmin, J. J. Luo and I. M. Daniel, "Processing of Expanded Graphite Reinforced Polymer Nanocomposites," Composites Science and Technology, Vol. 66, No. 9, 2006, pp. 1179-1186. doi:10.1016/j.compscitech.2005.10.014

[6] I. M. Afanasov, V. A. Morozov, A. V. Kepman, S. G. Ionov, A. N. Seleznev, G. V. Tendeloo and V. V. Avdeev, "Preparation, Electrical and Thermal Properties of New Exfoliated Graphite-Based Composites," Carbon, Vol. 47, No. 1, 2009, pp. 263-270. doi:10.1016/j.carbon.2008.10.004

[7] W. Weng, G. Chen, D. Wu, X. Chen, J. Lu and P. J. Wang, "Fabrication and Characterization of Nylon 6/Foliated Graphite Electrically Conducting Nanocomposite," Polymer Science Part-B Polymer Physics, Vol. 42, No. 15, 2004, pp. 2844-2856. doi:10.1002/polb.20140

[8] M. Xiao, L. Sun, J. Liu, Y. Li and K. G. Gong, "Synthesis and Properties of Polystyrene/Graphite Nanocomposites," Polymer, Vol. 43, No. 8, 2002, pp. 2245-2248. doi:10.1016/S0032-3861(02)00022-8

[9] D. R. Dreyer, S. Park, C. W. Bielawski and R. S. Ruoff, "The Chemistry of Graphene Oxide," Chemical Society
Reviews, Vol. 39, No. 1, 2010, pp. 228-240. doi:10.1039/b917103g

[10] G. Chen, C. Wu, W. Weng, D. Wu and W. Yan, "Preparation of Polystyrene/Graphite Nanosheet Composite," Polymer, Vol. 44, No. 6, 2003, pp. 1781-1784. doi:10.1016/S0032-3861(03)00050-8

[11] Y. Yang, J. Wang, J. Zhang, J. Liu, X. Yang and H. Zhao, "Exfoliated Graphite Oxide Decorated by PDMAEMA Chains and Polymer Particles," Langmuir, Vol. 25, No. 19, 2009, pp. 11808-11814. doi:10.1021/la901441p

[12] M. J. McAllister, J. L. Li, D. H. Adamson, H. C. Schniepp, A. A. Abdala, J. Liu, M. Herrera-Alonso, D. L. Milius, R. Car, R. K. Prud'homme and I. A. Aksay, "Single Sheet Functionalized Graphene by Oxidation and Thermal Expansion of Graphite," Chemistry of Materials, Vol. 19, No. 18, 2007, pp. 4396-4404. doi: $10.1021 / \mathrm{cm} 0630800$

[13] S. Stankovich, D. A. Dikin, R. D. Piner, K. A. Kohlhaas, A. Kleinhammes, Y. Jia, Y. Wu, S. T. Nguyen and R. S. Ruoff, "Synthesis of Graphene-Based Nanosheets via Chemical Reduction of Exfoliated Graphite Oxide," Carbon, Vol. 45, No. 7, 2007, pp. 1558-1565. doi:10.1016/j.carbon.2007.02.034

[14] M. Inagaki and T. Suwa, "Pore Structure Analysis of Exfoliated Graphite Using Image Processing of Scanning Electron Micrographs," Carbon, Vol. 39, No. 6, 2001, pp. 915-920. doi:10.1016/S0008-6223(00)00199-8

[15] G. Chen, W. Weng, D. Wu, C. Wu, J. Lu, P. Wang and X. Chen, "Preparation and Characterization of Graphite Nanosheets from Ultrasonic Powdering Technique," Carbon, Vol. 42, No. 4, 2004, pp. 753-759. doi:10.1016/j.carbon.2003.12.074

[16] M. Nakahara and Y. Sanada, "FT-IR ATR Spectroscopy of The Edge Surface of Pyrolytic Graphite and Its Surface/PVC Interface," Journal of Materials Science, Vol. 30, No. 17, 1995, pp. 4363-4368. doi:10.1007/BF00361518

[17] H. K. Jeong, Y, P. Lee, M. H. Jin, E. S. Kim, J. J. Bae and Y. H. Lee, "Thermal Stability of Graphite oxide," Chemical Physics Letters, Vol. 470, No. 4-6, 2009, pp. 255258. doi:10.1016/j.cplett.2009.01.050

[18] L. Hubert and V. B. Jose, "Handbook of Plastics Analysis," Marcel Dekker Inc., New York, 2003. 